\title{
Metagenomic Insights into The Diversity and Functions of Microbial Assemblages in Tasik Kenyir Ecosystem.
}

Mohd Ezhar Mohd Noor ${ }^{1}$, Sharifah Noor Emilia Syed Jamil Fadaak ${ }^{1}$, Mohd Noor Mat Isa ${ }^{2}$, Mohd Faizal Abu Bakar² \& Muhd Danish-Daniel Abdullah*1

${ }^{1}$ School of Fisheries and Aquaculture Science, Universiti Malaysia Terengganu, 21030 Kuala Terengganu, Terengganu, Malaysia.

${ }^{2}$ Malaysia Genome Institute, Ministry of Science, Technology and Innovation, Jalan Bangi, 43000 Kajang, Selangor, Malaysia.

Short Running Title: Metagenomic Analysis

Postal Address: School of Fisheries and Aquaculture Science, Universiti Malaysia Terengganu, 21030 Kuala Terengganu, Terengganu, Malaysia. (017-7231255)

Type of Paper: Original

Corresponding author email: mdda@umt.edu.my

Total Number of

Tables: 2

Figures: 4

Pages: 16 


\title{
Metagenomic Insights into The Diversity and Functions of Microbial Assemblages in
}

\author{
Tasik Kenyir Ecosystem.
}

\begin{abstract}
Tropical freshwater lake such as Tasik Kenyir are underrepresented among the growing number of environmental metagenomic data sets. In Tasik Kenyir, water from two different sites, pristine and disturbed areas were sampled. After the filtration process, genomic DNA from both sites were extracted using Meta-G-nome DNA isolation kit and shotgun metagenomic sequencing was carried out on Illumina HiSeq2500 Desktop Sequencer (Illumina, Inc.). Raw data were then trimmed and assembled using Metagenomic Assembler program, MetaVelvet. Data analysis was carried out using software Blast2GO (BioBam Bioinformatic S.L). The total number of sequence reads was 189,158 from TKS1.5m (disturbed area) and 246,577 from TKS2.5m (pristine area).The results indicate that sequence reads of microbial species were presence at disturbed area near the aquaculture zone was lower than the sequence reads of microbial species were presence at pristine area. When compared to archaea, both samples were dominated by bacteria (more than 90\%) suggesting that bacteria are absolutely dominant in the prokaryotic communities in the freshwater samples. The lake appears to contain a mixture of autotrophs and heterotrophs capable of performing main biogeochemical cycles like nitrogen fixation by Klebsiella sp for TKS1.5m and Pontibacter sp. for TKS2.5m. and carbon fixation by heterotrophic Alcaligenes sp. and Shewanella decolorationi in TKS1.5m, and by Pantoea sp. in TKS2.5m. Present study will advance our understanding of the importance of freshwater microbial communities for ecosystem and human health.
\end{abstract}

Keywords: metagenomic, microbial diversity, Tasik Kenyir 


\section{Introduction}

All biogeochemical cycles on biosphere involve with the microbes and major redox reactions such as the carbon cycle is catalyzed by a set of key microbial enzymes (Falkowski et al., 2008). Microbes generally can be found everywhere in the biosphere, including soil, water bodies and sediments. Szabo-Taylor et al., (2010) stated that microbes are widely known for their part in controlling the ecosystem function and biogeochemical cycling in the biosphere. It has been proposed by Newman and Banfield, (2002) that naturally, most microbes live under either nutrient- or energy-limiting conditions and due to this, they need to exist and blend in complex communities with the other microorganisms within the environments in order to survive.

Freshwater bodies such as Tasik Kenyir provide various ecological habitats and environment resources to flora and fauna. According to Portal of Lake Kenyir, (2014), Tasik Kenyir is the largest man-made lake in South East Asia and holds around 23.6 million cubic meters of water and occupies about 38000 hectares and also serves as another gateway to Malaysia National Park. Previous study by Amann et al., (1995) suggested that approximately less than $1 \%$ of earth's microorganisms are cultivable. In addition, Rinke et $a l .$, (2013) also reported the majority of the cultivated and genome-sequenced bacteria belong to only four phyla (Proteobacteria, Firmicutes, Actinobacteria, and Bacteroidetes). Meanwhile, recent data had suggested the existence of at least 63 bacterial phyla and candidate groups (SILVA database version 115) as reported by Pruesse et al., (2007) and Quast et al., (2013). These studies has given the researcher a better insight on the undiscovered genetic potential concealed by bacteria and archaea that evolved in billions of 
years. Therefore, a metagenomic analysis was done in order to compare the community compositions in Kenyir Lake as well as their role and functions.

Metagenomics is the technique used to extract the total genomic DNA of microorganisms directly from the environment and will represents the vast majority of microorganisms on earth as stated by Hugenholtz et al., (1998). The new generation of sequencing technology, with its ability to sequence thousands of organisms in parallel, has proved to be uniquely suited to this application. Metagenomics can be considered a revolutionary approach to study the microbial community that is unapproachable by available conventional methods and this approach also can capture the total genomes that present in a community of interest. As stated by Schloss and Handelsman, (2003), metagenomic was builds on advances in microbial genomics and in the polymerase chain reaction (PCR) amplification and cloning of genes. The field of metagenomic has play a major roles for in significant progress onmicrobial ecology, evolution, and diversity over the past 5 to 10 years. This development has opened new ways of understanding microbial diversity and functions. Hence, the characterization of the microbial community of Tasik Kenyir has potential implications for human activity and health which may apply to other freshwater lakes.

\section{Materials and Methods}

\section{Water Sampling}

Water samples were collected on January 2015 from Sungai Lasir (pristine area; N0459.997 E102 ${ }^{\circ} 50.133$ ) Sungai Como (disturbed area; N05 01.887 E102 50.600 ) i.e a transect between Mentong River and Terenggan River and another transect between Como River Aquaculture Zone and Sultan Mahmud Hydroelectricity Power Plant. Water from these areas were collected at the surface level, approximately 5 meters from the water surface. 
About $500 \mathrm{~mL}$ to $10 \mathrm{~L}$ of water were filtered through a filter cartridge. A membrane filter tower was constructed from polycarbonate filtration units (Millipore) with a $0.22 \mu \mathrm{m}$ pore size membrane filter (Millipore) aseptically placed on the filter holder. Membrane filters were sterilized by autoclaving. After filtration, samples were snap-frozen immediately in liquid nitrogen and stored in $-80^{\circ} \mathrm{C}$ until further use.

\section{Metagenomic Analysis}

Nucleic acid extractions were performed by using Metagenome DNA isolation kit (Epicentre) and PowerWater DNA Isolation Kit (MO BIO Lab. Inc.). After that, gel Electrophoresis and Documentation was run to check the Quality and Quantity of the DNA. Shotgun metagenomic sequencing was carried out on Illumina HiSeq2500 Desktop Sequencer (Illumina, Inc.). The sequencing was performed by Macrogen Inc., Korea. Illumina platform was chosen due to lower cost and limited systematic errors compared to other platform. The generated multi-million reads were then trimmed and assembled by Metagenomic Assembler program, MetaVelvet. Data analysis was carried out using software Blast2GO (BioBam Bioinformatic S.L). Then initial taxonomic analysis was performed by bioinformatic tools like BLAST (Altschul et al. 1997) and MEGAN (Huson et al. 2007).

\section{Results and Discussion}

Sequence reads data from two sites were collectively used to analyze microbial diversity. The total number of reads was 189,158 from TKS1.5m (disturbed area) and 246,577 from TKS2.5m (pristine area) (Table 1). When compared to Archaea, both samples were dominated by Bacteria with more than $90 \%$, suggesting that bacteria are absolutely dominant in the prokaryotic communities in the freshwater samples. Phylogenetic analysis of 
both samples also revealed that the overwhelming majority of the data set were bacterial and archaeal. Most of the archaeal metagenomics reads from TKS1.5m area matched Methanosaeta thermophile with a few additional representatives within Thermococci while archaeal metagenomics reads from TKS2.5m area matched Methanolobus psychrophilus. Both metagenomics data sets agreed with the broad taxonomic picture that almost $90 \%$ of the sequences affiliated to Bacteria, whereas Archaea were a minor component. The remaining about $10 \%$ sequences were of eukaryotic origin and virus. All major bacterial groups commonly found in freshwater ecosystems that has been reported by Humbert et al., (2009) were present in Tasik Kenyir.

Shotgun metagenomic analysis revealed that both samples were dominated by Proteobacteria (more than $65 \%$, Table 2). The finding is consistent with findings of past studies by Kersters et al., (2006), which reported that Proteobacteria, the largest and most phenotypically diverse phylum, accounting for at least $40 \%$ of all known genera. In addition, present study, phyla in common to all TKS1.5m and TKS $2.5 \mathrm{~m}$ samples (Verrucomicrobia, Bacteroidetes, and Firmicutes, Cyanobacteria; Table 2) can be found in a variety of aquatic environments similar to the finding by Dillon et al., (2009) although latter phyla were far less dominant than the Proteobacteria. In contrast to sample fom pristine area, TKS2.5m, sample from disturbed area (aquaculture site), TKS1.5m was dominated by pathogenic bacteria species like Pseudomonas species, Aeromonas species, Klebsiella species and Vibrio species (Figure 3). Austin and Austin (1999) stated that common fish pathogenic bacterial species belong to the genera Vibrio, Aeromonas, Flavobacterium, Yersinia, Edwardsiella and Pseudomonas. In addtion, study by Martin-Carnahan and Joseph (2005) also stated that Aeromonads are among the most common bacteria in aquatic environments and are frequently associated with severe diseases among cultured fishes . 
As known, Bacteria in the genus Vibrio are mainly pathogenic to marine and brakishwater fish. However, the scientific report by Lightner and Redman (1998) stated they are occasionally reported in freshwater species. Research finding by Actis et al., (2011) also points toward that vibriosis, one of the major bacterial diseases affecting fish is primarily caused by pathogenic species such as Vibrio harveyii, V. anguillarum and V. ordalii. These vibrios can be found abundantly in the sample from aquaculture site, TKS1.5m compared to pristine area sample, TKS2.5m. Although pathogenic species representing majority of existing bacterial taxa, only a relatively small number of pathogens are responsible for important economic losses in cultured fish worldwide. Present result analysis also revealed that microbial community from TKS $2.5 \mathrm{~m}$ was more diverse than the TKS $1.5 \mathrm{~m}$. Consistent with findings by Kennedy (1999), the diversity of microbial is critical to the functioning of the ecosystem, because there is the need to maintain ecological processes like controlling pathogens within the ecosystems. Study by Yamanaka et al. (2003) also stated that microbial diversity is directly related to ecosystem stability.

As Bacteria and Archaea accounted for most of total metagenomic reads, the comparative study of the geochemistry of carbon $(\mathrm{C})$ and nitrogen $(\mathrm{N})$ will be focused on the prokaryotes. For the carbon cycling, the pathway detected in the oxic-anoxic interface was aerobic respiration by heterotrophic Alcaligenes sp. from the order Burkholderiales and Shewanella decolorationi from Alteromonadales in TKS1.5m, and by Pantoea sp. from Enterobacteriales in TKS $2.5 \mathrm{~m}$. Carbon cycling is a key metabolic feature of members of the alpha and gamma Proteobacteria, including Alteromonadaceae, Pseudomonadaceae, Sphingomonadaceae, and Vibrionaceae as well as Flavobacteriaceae, which is member of the Cytophaga-Flavobacteria-Bacteroides (CFB) clade. The present finding also support the 
previous studies by Ramaiah et al., (2000), Ivanova and Mikhailov, (2001), Kirchman, (2002) Kirchman et al., (2004) and Mikhailov et al., (2006) which concluded that these bacteria produce enzymes capable of degrading various polysaccharides, such as cellulose, chitin, and pectin. For the nitrogen cycle, most of the detected marker genes catalyzed Nitrogen fixation by Klebsiella sp for TKS1.5m and Pontibacter sp. for TKS2.5m. The result is in the lines of earlier literatures by Jenni et al., (1989), Willems et al., (1991) and Chimetto et al., (2008) which found that nitrogen fixation has been reported among members of the Comamonadaceae, Pseudomonadaceae, and Vibrionaceae families. Meanwhile, denitrification was observed in relatively low abundance in both samples but, metagenomic reads matching Nitrospirae nitrite-oxidizing bacteria were detected in both samples, pointing out that the genetic potential to close the nitrogen cycle was there. Studies by Paerl et al., (2002) and Rabalais et al., (2009) stated denitrification is important in aquatic environments because a build-up of nitrate can lead to eutrophication and harmful algal blooms. Earlier studies by Cole, (1996), Chou et. al., (2008) and Rajakumar et al., (2008) showed that nitrogen cycling is the key metabolic trait shared by different bacteria associated with both samples where many of the bacteria, including members of the Comamonadaceae, Enterobacteriaceae and Pseudomonadaceae, can reduce nitrate.

\section{Conclusion}

It is known in present study that prokaryote community bacteria is absolutely dominant over archaea with more than $90 \%$ in metagenomic pools in the freshwater prokaryotic samples. Tasik Kenyir metagenomic data sets provides the means to examine with fine-scale resolution how natural microbial populations are impacted by human activities (aquaculture site) and major environmental perturbations. Hence, it enable researchers to identify some of the major microbial players and functions that could be targets for future 
work examining the response and resilience of these communities to environmental changes and human perturbations. Such studies will significantly advance the understanding of the importance of microbial communities for ecosystem and human health especially in freshwater environment.

\section{Acknowledgements}

This research was funded by the Ministry of Higher Education Malaysia through grant FRGS 2014 Vot No. 59334. We thanks all the people involved directly or indirectly in this study especially staffs from the School of Fisheries and Aquaculture Sciences, UMT, Mr Sharol Ali and senior scientist from Malaysia Genome Institute, Mr. Faizal Abu Bakar.

\section{References}

Actis, L. A., Tolmasky, M. E. and Crosa, J. H. Vibriosis in Fish Diseases and Disorders, Viral, Bacterial and Fungal Infections Woo, P. T. K. and Bruno, D. W. Eds. 3, 570-605 (2011).

Altschul, S. F., Thomas L. M., Alejandro A. S., Jinghui, Z., Zheng, Z., Webb, M. and David J. L: Gapped BLAST and PSI-BLAST: a new generation of protein database search programs. Nucleic. Acids. Res., 25, 3389-3402 (1997).

Amann, R. I., Ludwig, W. and Schleifer K. H: Phylogenetic identification and in situ detection of individual microbial cells without cultivation. Microbiol. Rev., 59, 143169 (1995).

Austin, B. and Austin, D. A. bacterial fish pathogens: diseases in farmed and wild fish. Springer, New York, NY, USA. 3rd Edition (1999). 
Brock, T. D: The study of microorganisms in situ: progress and problems. Symp. Soc. Gen. Microbiol., 41, 1-17 (1987).

Chimetto, L. A., Brocchi, M., Thompson, C. C., Martins, R. C. R., Ramos, H. R. and Thompson, F. L: Vibrios dominate as culturable nitrogen-fixing bacteria of the Brazillian coral Mussismilia hispida. Syst. Appl. Microbiol., 31, 312-319 (2008).

Chou, Y. J., Chou, J. H., Lin, K. Y., Lin, M. C., Wei, Y.H., Arun, A. B., Young, C. C. and Chen, W.M: Rothia terrae sp. nov. isolated from soil in Taiwan. Int. J. Syst. Evol. Microbiol., 58, 84-88 (2008).

Cole, J.: Nitrate reduction to ammonia by enteric bacteria: redundancy, or a strategy for survival during oxygen starvation?. FEMS. Microbiol. Lett., 136, 1-11 (1996).

Dillon, J. G., McMath, L. M., and Trout, A. L: Seasonal changes in bacterial diversity in the Salton Sea. Hydrobiologia., 632, 49-56 (2009).

Falkowski, P. G., Fenchel, T. and DeLong, E. F: The microbial engine that drive Earth's biogeochemical cycles. Science., 320, 1034-1039 (2008).

Hugenholtz, P., Goebel, B. M. and Pace, N. R: Impact of culture-independent studies on the emerging phylogenetic view of bacterial diversity. J. Bacteriol., 180, 4765-4774 (1998).

Humbert, J.-F., Dorigo, U., Cecchi, P., Le Berre, B., Debroas, D. and Bouvy, M.: Comparison of the structure and composition of bacterial communities from temperate and tropical freshwater ecosystems. Environ. Microbiol., 11, 2339-2350 (2009).

Huson. D. H, Auch. A.F, Qi. J, and Schuster. S. C: MEGAN analysis of metagenomic data. Genome. Res., 17, 377-386 (2007). 
Ivanova, E. P., and Mikhailov, V. V.: A new family, Alteromonadaceae fam. nov. including marine proteobacteria of the genera Alteromonas, Pseudoalteromonas, Idiomarina, and Colwellia. Microbiol., 70, 10-17 (2001).

Jenni, B., Isch, C., and Aragno, M.: Nitrogen fixation by new strains of Pseudomonas pseudoflava and related bacteria. Microbiol., 135, 461-467 (1989).

Kennedy, A. C. Bacterial diversity in agroecosystems. Agr Ecosyst Environ. 74 (1), 65-76 (1999)

Kersters, K., De Vos, P., Gillis, M., Swings, J. Vandamme, P. and Stackebrandt, E.: Introduction to the Proteobacteria. In: The Prokaryotes. (Eds.: Dworkin, M., Falkow, S., Rosenberg, E., Schleifer, K. H. and Stackebrandt, E.) Springer, New York., p. 3-37 (2006).

Kirchman, D. L.: The ecology of Cytophaga-Flavobacteria in aquatic environments. FEMS Microbiol. Ecol., 39, 91-100 (2002).

Kirchman, D. L., Ditteh, A. I., Findlay, S. E. G. and Fischer, D.: Changes in bacterial activity and community structure in response to dissolved organic matter in the Hudson River, New York. Aquat. Microb. Ecol., 35, 243-257 (2004).

Lightner, D. V. and Redman, R. M. Shrimp diseases and current diagnostic methods. Aqua. 164, 201-220 (1998).

Martin-Carnahan, A. and Joseph, S. W. Genus I. Aeromonas Stanier 1943, 213AL. In Bergey's Manual of Systematic Bacteriology, 2nd edn, vol. 2, part B, pp. 557-578. Edited by D. J. Brenner, N. R. Krieg, J. T. Staley and G. M. Garrity. New York: Springer (2005)

Mikhailov, V., Romanenko, L., and Ivanova, E.: The genus Alteromonas and related proteobacteria. In: The Prokaryotes (Eds.: Dworkin, M., Falkow, S., Rosenberg, E., Schleifer, K. H. and Stackebrandt, E.) Springer, New York. p. 597-645 (2006). 
Newman, D. K. and Banfield, J. F.: Geomicrobiology: how molecular-scale interactions underpin biogeochemical systems. Science., 296, 1071-1077 (2002).

Paerl, H. W., Dennis, R. L., and Whitall, D. R.. Atmospheric deposition of nitrogen: implications for nutrient over-enrichment of coastal waters. Estuaries., 25, 677-693 (2002).

Portal of Kenyir Lake, 2014. Accessed on August 2014 www.kenyirlake.com.my.

Pruesse, E., Quast C., Knittel K., Fuchs B.M., and Ludwig, W.: SILVA: a comprehensive online resource for quality checked and aligned ribosomal RNA sequence data compatible with ARB. Nucleic. Acids. Res., 35, 7188-7196 (2007).

Quast, C., Pruesse E., Yilmaz P., Gerken J., and Schweer T.: The SILVA ribosomal RNA gene database project: improved data processing and web-based tools. Nucleic. Acids. Res., 41, 590-596 (2013).

Rabalais, N. N., Turner, R. E., Diaz, R. J., and Justıc, D.: Global change and eutrophication of coastal waters. ICES J. Mar. Sci., 66, 1528--537 (2009).

Rajakumar, S., Ayyasamy, P. M., Shanthi, K., Thavamani, P., Velmurugan, P., $\quad$ Song, $\quad$ Y. C., and Lakshmanaperumalsamy, P.: Nitrate removal efficiency of bacterial consortium (Pseudomonas sp. KW1 and Bacillus sp. YW4) in synthetic nitrate-rich water. J. Hazard. Mater., 157, 553-563 (2008).

Ramaiah, N., Hill, R. T., Chun, J., Ravel, J., Matte, M. H., Straube, W. L. and Colwell, R. R.: Use of a chi-A probe for detection of chitinase genes in bacteria from the Chesapeake Bay. FEMS Microbiol. Ecol., 34, 63-71 (2000).

Rinke, C., Schwientek P., Sczyrba A., Ivanova N. N. and Anderson I. J.: Insights into the phylogeny and coding potential of microbial dark matter. Nature., 499, 431-437 (2013). 
Schloss P.D. and Handelsman J.: Biotechnological prospects from metagenomics. Curr. Opin. Biotech., 14, 303-310 (2003).

Szabo-Taylor, K. E., Kiss, K. T., Logares, R., Eiler, A., Acs, E., Toth, B. and Bertilsson, S.: Composition and dynamics of microeukaryote communities in the River Danube,Fottea. 10(1), 99-113 (2010).

Willems, A., J. De Ley, M. Gillis, and K. Kersters.: Comamonadaceae, a new family encompassing the Acidovorans rRNA complex, including Variovorax paradoxus gen. nov., comb. nov., for Alcaligenes paradoxus (Davis 1969). Int. J. Syst. Evol. Microbiol., 41, 445-450 (1991).

Yamanaka, T., Helgeland, L., Farstad, I. N., Fukushima, H., Midtvedt, T. and Brandtzaeg, P. Microbial colonization drives lymphocyte accumulation and differentiation in the follicle-associated epithelium of Peyer's patches. J. Immunol. 170(2), 816-822 (2003).

\section{List of Tables}

Table 1. Characteristics of shotgun metagenomic libraries

\section{Sample designation}

Characteristic

TKS1.5m

189158

66338

4153
TKS2.5m

246577

Genes Reads

Species Detected
106097

32421 
Table 2. Percentage of Domains and bacterial phyla from TKS $1.5 \mathrm{~m}$ and TKS2.5m

Sample designation

Domains/Phyla (Bacteria)

TKS1.5m

TKS $2.5 \mathrm{~m}$

\begin{tabular}{ccc}
\hline Proteobacteria & $(65 \%)$ & $(72 \%)$ \\
Bacteroidetes & $(5 \%)$ & $(3 \%)$ \\
Cyanobacteria & $(1 \%)$ & $(1 \%)$ \\
Firmicutes & $(8 \%)$ & $(5 \%)$ \\
Verrucomicrobia & $(5 \%)$ & $(4 \%)$ \\
Other Phyla & $(8 \%)$ & $(2 \%)$ \\
Unclassified & $(3 \%)$ & $(2 \%)$ \\
Eucarya & $(3 \%)$ & $(2 \%)$ \\
Archaea & $(1 \%)$ & $(1 \%)$ \\
Virus & $(1 \%)$ & $(8 \%)$ \\
\hline
\end{tabular}

\section{List of Figures}

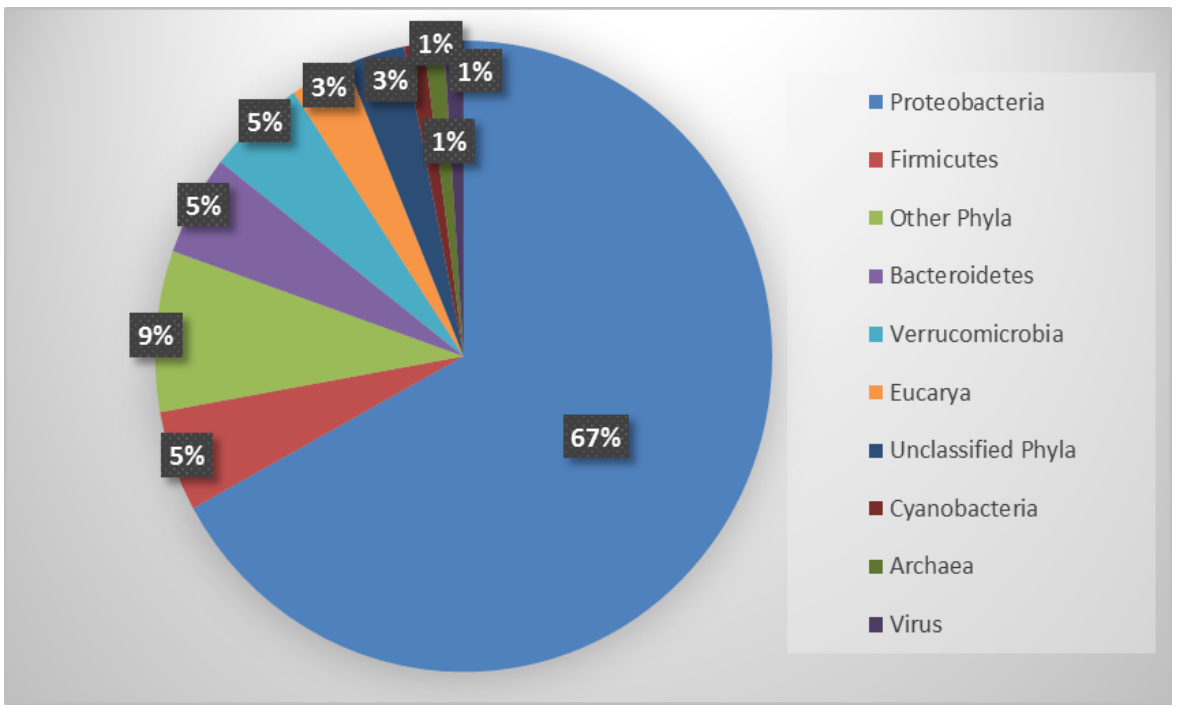

Figure 1: Relative abundance of the domain and dominant bacterial phyla from TKS1.5m 


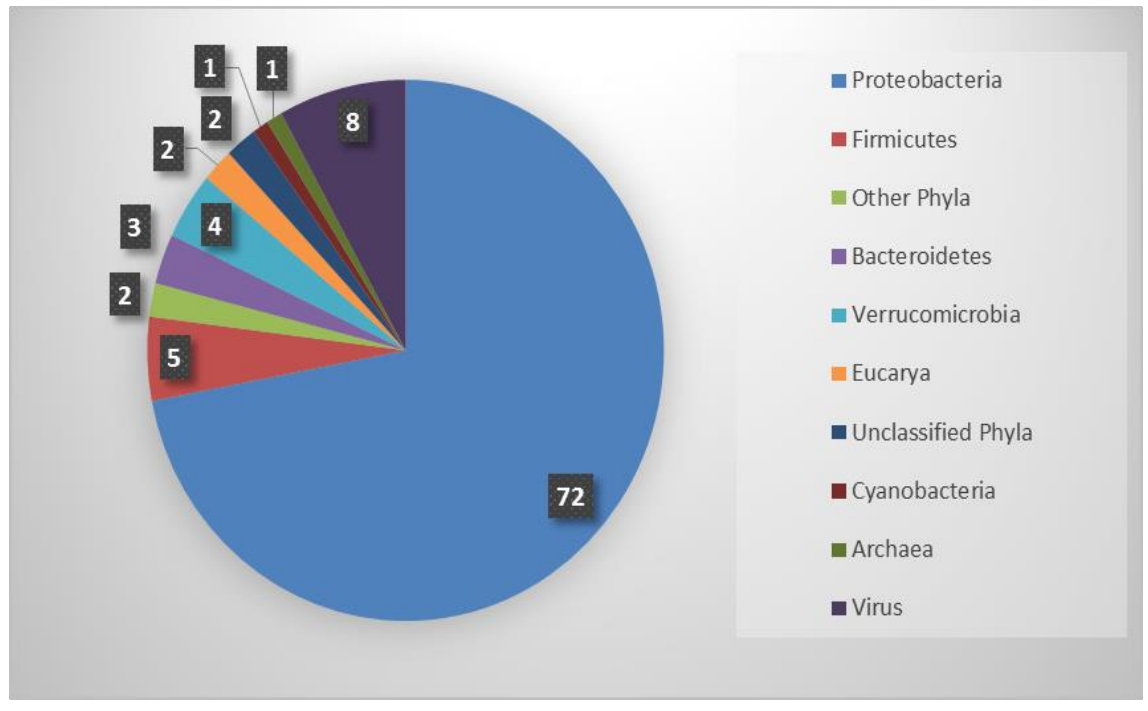

Figure 2: Relative abundance of the domain and dominant bacterial phyla from TKS2.5m.

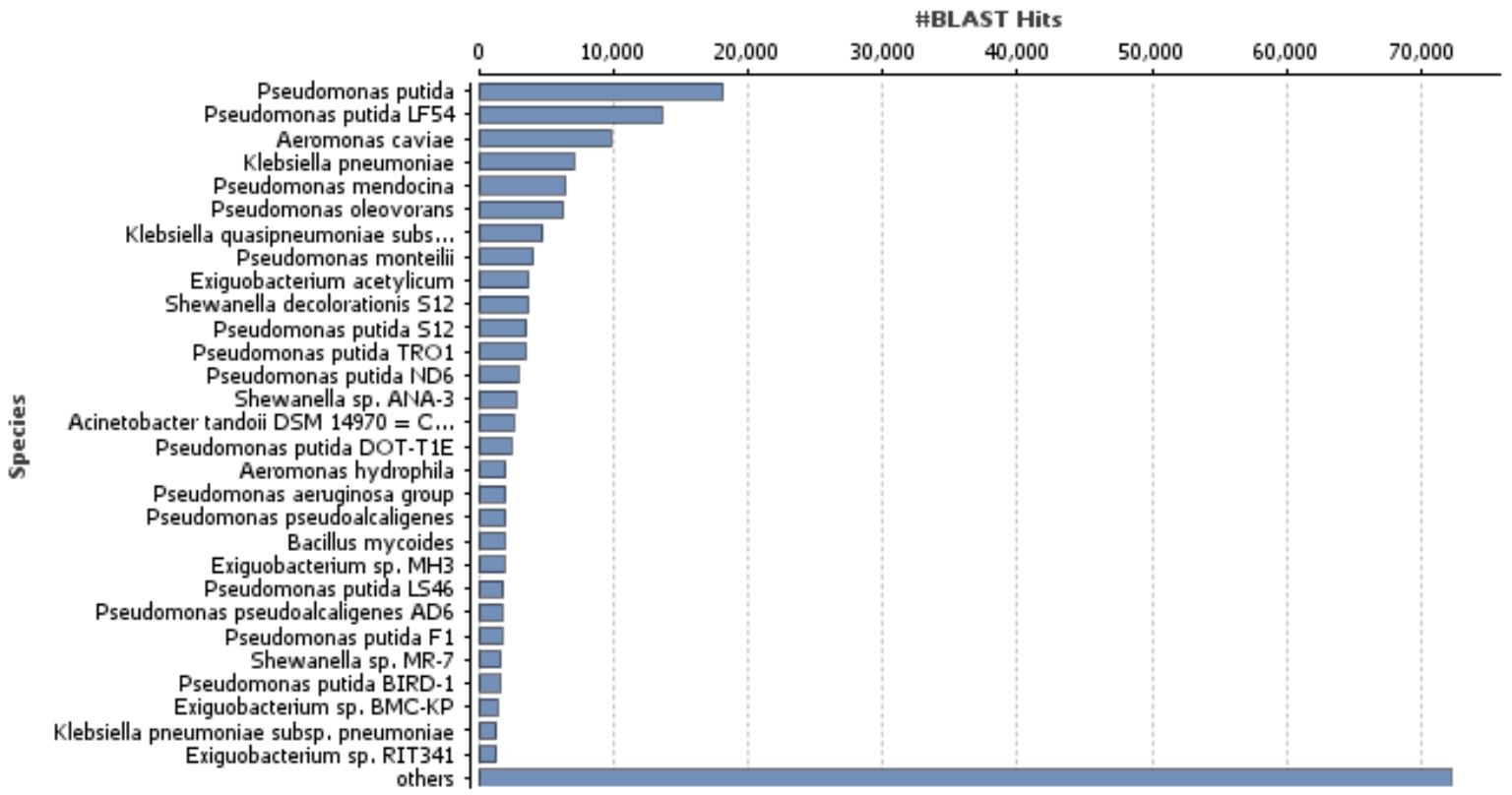

Figure 3: Species Distribution of microbial community detected from the TKS1.5m (Aquaculture Area) metagenome sequences. 


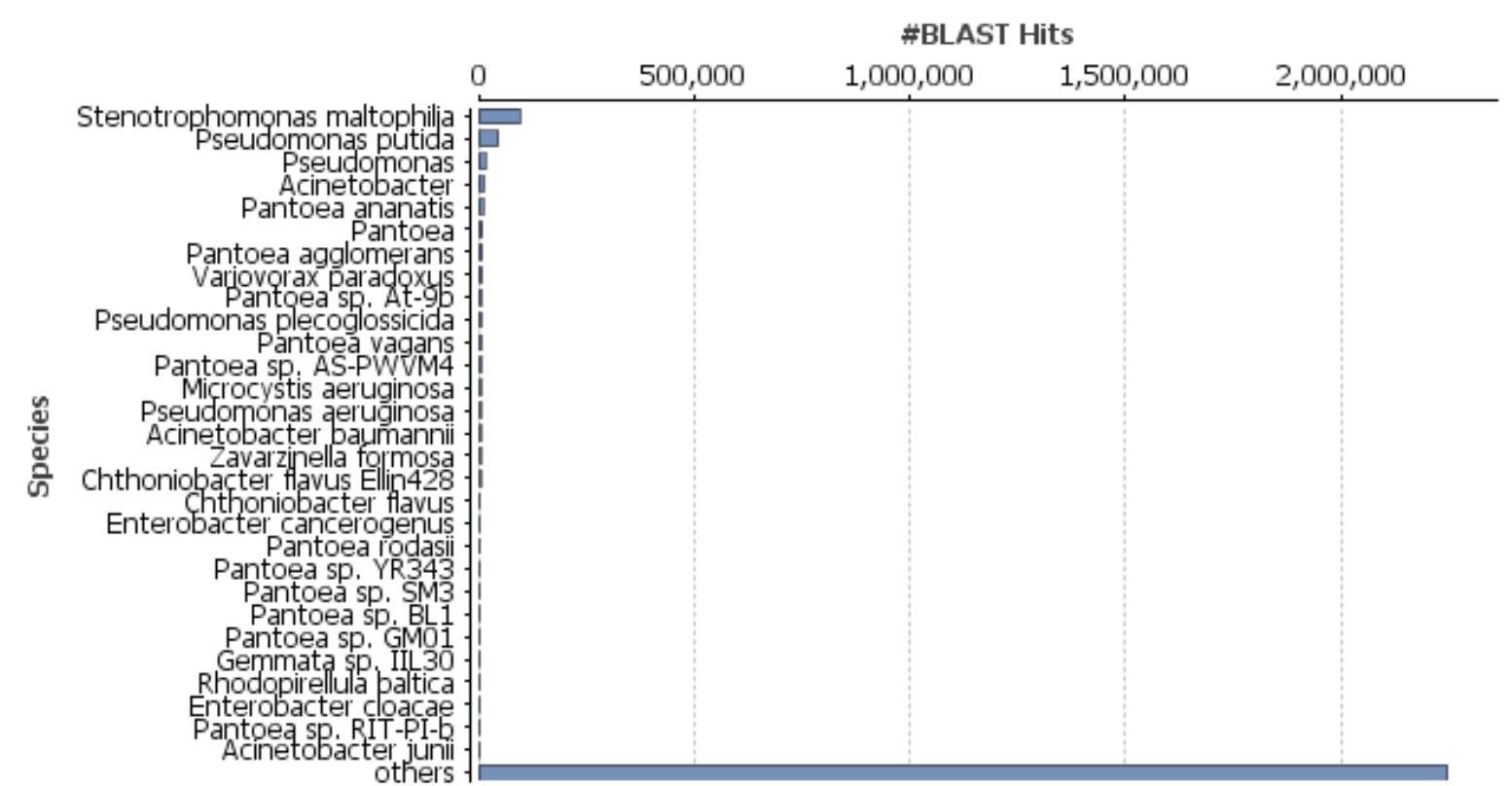

Figure 4: Species Distribution of microbial community detected from the TKS2.5m (Pristine Area) metagenome sequences. 